\title{
Treatment of Diastolic Heart Failure in Hypertensive Diabetic Patient - Between Illusion and Achievements
}

\author{
Sur Genel ${ }^{1,2 *}$, Floca Emanuela ${ }^{1}$, Sur M Lucia ${ }^{1}$, Sur G Daniel ${ }^{1}$ and Radulescu Dan ${ }^{1,3}$ \\ ${ }^{1}$ University of Medicine and Pharmacy, Iuliu Hatieganu, Cluj-Napoca, Romania \\ ${ }^{2}$ Emergency Clinical Hospital for Children, Cluj-Napoca, Romania \\ ${ }^{3}$ Department of Cardiology, Municipal Hospital Cluj Napoca, Romania
}

\begin{abstract}
Hypertension is the most prevalent cardiovascular disease in the world. Because of associated morbidity and mortality it is in one of the most important public health problems. Hypertension is the most important cause of heart failure with low or preserved ejection fraction. If hypertension develops concomitant with diabetes mellitus treatment problem of the two diseases becomes more complex. It is known that beta-blockers may induce type 2 diabetes, but those of new generation such as Nebivolol do not have this effect.
\end{abstract}

There are many drugs with proven efficacy in lowering blood pressure, but the optimal treatment to prevent progression to heart failure is uncertain. Beta-blockers are a class of drugs with benefits both on hypertension and in heart failure. Drugs of this class have different pharmacological properties in terms hemodynamic and cardiovascular.

Nebivolol is a beta-blocker that causes vasodilatation mediated by nitric oxide release. This medicine lowers blood pressure, prevents endothelial dysfunction and improves coronary flow reserve and diastolic function independent of ventricular geometry changes. Action of nebivolol is superior to classic beta blockers by reversibility of subclinical changes in the left ventricle before the onset of heart failure.

In the early stages of heart failure with preserved ejection fraction the management is not yet established. Therefore it is important to know that in these situations nebivolol has beneficial effects.

Keywords: Diastolic; Heart failure; Nebivolol; Diabetes; Hypertension

\section{Introduction}

There is a high prevalence of hypertension worldwide. Prevalence is higher for males until the age of 50 years. There is an increasing prevalence of this pathology along with age. Hypertension and diabetes are the main cause of heart failure with the preservation or decreased of the ejection fraction thus representing an important public health problem. [1]

\section{How Does Diastolic Heart Failure}

In patients with hypertension structural changes both cardiac and vascular occur as a consequence of increasing blood pressure. At the same time, these structural changes develop in an attempt to normalize wall stress. Cardiac remodeling has functional consequences by which cardiovascular risk is increased. These consequences are related to the individual aspects such as age, 24 hours blood pressure, the rigidity of blood pressure, plasma volume, neuro-hormonal status, genetic aspects $[2,3]$.

Ventricular hypertrophy involves both myocytes and interstitial tissue. Interstitial tissue can lead to fibrosis, a phenomenon that contributes to cardiac dysfunction in hypertension. The neuroendocrine changes that occur with aging such as decreased b-adrenergic receptor density, decreased b-adrenergic inotropic response, and increased angiotensin receptors and angiotensinogen and angiotensin-converting enzyme concentrations contributes to myocyte hypertrophy. Hypertrophy and fibrosis of left ventricle reduce ventricular compliance finally leading to diastolic failure [4-6].

There are numerous studies showing that arterial stiffness is increased in hypertension. Vascular stiffness influences the propagation velocity of pressure wave generated by cardiac contraction. Pressure wave transmitted through the vessels back to the heart in a short time, resulting in increased pressure of anterograde wave and decreased blood flow. Aortic compliance is low $[4,6]$. Wave reflection can be accelerated, increasing left ventricular ejection resistance. This mechanism contributes to left ventricular hypertrophy which is associated with impaired diastolic function. Arterial stiffness contributes to myocardial ischemia by altering ventriculo-arterial coupling. In patients with hypertension microvascular ischemia and interstitial fibrosis determine subendocardial dysfunction $[7,8]$.

Regarding myocardial architecture some studies have shown that shortening in the fibers of longitudinal axis is followed by shortening in the circumferential axis. Longitudinal shortening plays a role in contractile function of the heart being involved in ventricular ejection [6].

Diabetes contributes to the development of heart failure by excessive myocardial fibrosis, interstitial accumulation of glycoproteins, and an altered release from dysfunctional coronary endothelium of mediators such as nitric oxide which has vascular relaxation effect $[9,10]$.

\section{How to Diagnose Diastolic Heart Failure}

A diagnosis of diastolic heart failure requires the mandatory

${ }^{*}$ Corresponding author: Sur Genel, University of Medicine and Pharmacy, Iuliu Hatieganu, Cluj-Napoca, Romania, Tel: 0724504964; E-mail: surgenel@yahoo.com

Received February 07, 2014; Accepted February 12, 2014; Published February 17,2014

Citation: Genel S, Emanuela F, Lucia SM, Daniel SG, Dan R (2014) Treatment of Diastolic Heart Failure in Hypertensive Diabetic Patient - Between Illusion and Achievements. J Diabetes Metab 5: e113. doi:10.4172/2155-6156.1000e113

Copyright: ( 2014 Genel S, et al. This is an open-access article distributed under the terms of the Creative Commons Attribution License, which permits unrestricted use, distribution, and reproduction in any medium, provided the original author and source are credited. 
presence of three criteria: (1) presence of signs or symptoms of congestive heart failure; (2) presence of normal or midly reduced left ventricular systolic function; (3) evidence of abnormal left ventricular relaxation, filling, diastolic distensibility or diastolic stiffness $[2,8]$.

Signs and symptoms of congestive heart failure include paroxysmal nocturnal dyspnea, orthopnea, gallop sounds, lung crepitations, pulmonary edema, and peripheral edema [3].

A diagnosis of diastolic heart failure requires the presence of normal or midly abnormal left ventricular systolic function. To meet this criterion ventricular ejection fraction must be at least $45 \%$. Left ventricular relaxation and filling affect left ventricular diastolic distensibility. So diagnostic of diastolic heart failure can be obtained from analysis of indices of diastolic function such as relaxation, chamber and myocardial stiffness, diastolic filling characteristics. This analysis can be performed using invasive or noninvasive techniques. Thus echo-Doppler studies can provide information about relaxation (isovolumic relaxation time), abnormalities of filling and changes in left ventricular diastolic pressure [5].

\section{How to Treat Diastolic Heart Failure}

Treatment of diastolic heart failure is not yet well documented. Nevertheless there are studies that attempt to show the role of Nebivolol in the treatment of diastolic heart failure $[1,3]$.

By the action of nitric oxide release Nebivolol is one of the most powerful lusitropic agents with additional vasodilating properties. Thus Nebivolol has important therapeutic implications against the cardiovascular risk factors and especially on atherosclerosis [11].

Prospective randomized trials comparing the effects of Nebivolol and Atenolol in hypertensive patients with diastolic dysfunction showed that Nebivolol improves haemodynamic status both at rest and stress. Nebivolol improves the diastolic function and lowers blood pressure and heart rate at rest and at peak stress; it also decreases ventricular mass. The good effect of Nebivolol versus Atenolol may be explained by secondary vasodilatory action of nitric oxide release $[12,13]$

It is known that drugs that increase the release of nitric oxide significantly reduce the arterial reflected wave. Other studies have shown that in hypertension nebivolol decreases not only aortic stiffness but also reflected wave and the centric aortic pulse pressure. But several studies have shown that early longitudinal diastolic function significantly increased only in patients treated with Nebivolol $[13,14]$. Nebivolol also significantly increased the longitudinal displacement and the ejection time, thus providing an improved hemodynamic profile. The latest beta-blockers such as Nebivolol improve filling pressure independently of the presence of left ventricular hypertrophy. Effects of Nebivolol on diastolic function are influenced by the duration of treatment and the potential release of nitric oxide. To achieve the desired effects duration of treatment must be at least 3 months $[11,12]$.

Regarding the metabolic effect Nebivolol has recently been shown not to worsen glucose tolerance compared with placebo. Hemodynamic profile of Nebivolol characterized by preserving cardiac output, ejection time prolongation, reduction of peripheral resistance and improved diastolic function, has relevant benefits on the impairment in diastolic function [15].

We followed 48 patients diagnosed with hypertension and diabetes who had left ventricular hypertrophy without coronary heart disease. They were treated for 6 months with Nebivolol and this treatment resulted in improved diastolic function.

\section{References}

1. The Task force for the management of arterial hypertension of the European Society of Hypertension (ESH) and the European Society of Cardiology (ESC) (2013) $2013 \mathrm{ESH} / \mathrm{ESC}$ Guidelines for the management of arterial hypertension. J Hypertens 31: 1281-1357.

2. McDonald K (2008) Diastolic heart failure in the elderly: underlying mechanisms and clinical relevance. Int J Cardiol 125: 197-202.

3. Shammas RL, Khan NU, Nekkanti R, Movahed A (2007) Diastolic heart failure and left ventricular diastolic dysfunction: what we know, and what we don't know! Int J Cardiol 115: 284-292.

4. Cheng S, Fernandes VR, Bluemke DA, McClelland RL, Kronmal RA, et al. (2009) Age-related left ventricular remodeling and associated risk for cardiovascular outcomes: the Multi-Ethnic Study of Atherosclerosis. Circ Cardiovasc Imaging 2: 191-198.

5. Gary R, Davis L (2008) Diastolic heart failure. Heart Lung 37: 405-416.

6. Kane GC, Karon BL, Mahoney DW, Redfield MM, Roger VL, et al. (2011) Progression of left ventricular diastolic dysfunction and risk of heart failure. JAMA 306: 856-863.

7. Brahmajee K Nallamothu, Timir S Baman, Anubhav Garg, Scott L Hummel (2013) Inpatient Cardiovascular Medicine.

8. Borlaug BA, Redfield MM (2011) Diastolic and systolic heart failure are distinct phenotypes within the heart failure spectrum. Circulation 123: 2006-2013.

9. From AM, Scott CG, Chen HH (2010) The development of heart failure in patients with diabetes mellitus and pre-clinical diastolic dysfunction a population-based study. J Am Coll Cardiol 55: 300-305.

10. Tribouilloy $C$, Rusinaru $D$, Mahjoub $H$, Tartière $J M$, Kesri-Tartière $L$, et al. (2008) Prognostic impact of diabetes mellitus in patients with heart failure and preserved ejection fraction: a prospective five-year study. Heart 94: 1450-1455

11. Conraads VM, Metra M, Kamp O, De Keulenaer GW, Pieske B, et al. (2011) Effects of the long-term administration of nebivolol on the clinical symptoms, exercise capacity, and left ventricular function of patients with diastolic dysfunction: results of the ELANDD study. European Journal of Heart Failure 14: $219-225$

12. Zhou X, Ma L, Habibi J, Whaley-Connell A, Hayden MR, et al. () Nebivolo Improves Diastolic Dysfunction and Myocardial Remodeling Through Reductions in Oxidative Stress in the Zucker Obese Rat. Hypertension 55: 880-888.

13. Vinereanu D, Gherghinescu C, Ciobanu AO, Magda S, Niculescu N, et al. (2011) Reversal of subclinical left ventricular dysfunction by antihypertensive treatment: a prospective trial of nebivolol against metoprolol. J Hypertens 29: 809-817.

14. Fang Y, Nicol L, Harouki N, Monteil C, Wecker D, et al. (2011) Improvement of left ventricular diastolic function induced by $\hat{~}^{2}$-blockade: a comparison between nebivolol and metoprolol. J Mol Cell Cardiol 51: 168-176.

15. Ma L, Gul R, Habibi J, Yang M, Pulakat L, et al. (2012) Nebivolol improves diastolic dysfunction and myocardial remodeling through reductions in oxidative stress in the transgenic (mRen2) rat. Am J Physiol Heart Circ Physiol 302 2341-2351. 